\title{
Development and validation of nomograms for predicting survival and recurrence in patients with parotid gland cancer
}

\author{
Zhigong Wei ${ }^{1 \#}$, Zhuang Zhang ${ }^{2 \#}$, Lianlian Yang ${ }^{1}$, Ling He ${ }^{1}$, Zheran Liu ${ }^{1}$, Yan He ${ }^{1}$, Jingjing Wang ${ }^{1}$, \\ Xiaoli Mu ${ }^{1}$, Ruidan $\mathrm{Li}^{1}$, Yan Huang ${ }^{1}$, Xingchen Peng ${ }^{1}$ \\ ${ }^{1}$ Department of Biotherapy, Cancer Center, West China Hospital, Sichuan University, Chengdu, China; ${ }^{2}$ State Key Laboratory of Oral Diseases, \\ National Clinical Research Center for Oral Diseases, Department of Head and Neck Oncology, West China Hospital of Stomatology, Sichuan \\ University, Chengdu, China \\ Contributions: (I) Conception and design: X Peng, Z Wei; (II) Administrative support: X Peng, Z Zhang; (III) Provision of study materials or patients: \\ X Peng, Z Zhang; (IV) Collection and assembly of data: Z Wei, L Yang, L He, Z Liu, Y He, J Wang, X Mu, R Li, Y Huang; (V) Data analysis and \\ interpretation: Z Wei, Z Zhang, L Yang, L He; (VI) Manuscript writing: All authors; (VII) Final approval of manuscript: All authors. \\ \#These authors contributed equally to this work. \\ Correspondence to: Xingchen Peng. Department of Biotherapy, Cancer Center, West China Hospital, Sichuan University, No.37 Guoxue Alley, Wuhou \\ District, Chengdu 610041, China. Email: pxx2014@163.com.
}

Background: To develop nomograms for predicting recurrence risk and long-term survival in patients with parotid gland cancer (PGC).

Methods: A total of 301 consecutive patients with PGC who underwent surgery were enrolled and randomly divided into a training cohort $(n=210)$ and a validation cohort $(n=91)$. Predictive nomograms were constructed based on the independent indicators of overall survival (OS) and disease-free survival (DFS) as determined by multivariate Cox regression analysis. The discrimination and calibration of nomograms were evaluated using C-indices and calibration curves.

Results: Six independent predictors of OS were identified. Incorporating these factors, the nomogram showed good concordance statistics of 0.84 and 0.78 in predicting the 5 -year OS in the training and validation cohorts. Five independent predictors of DFS were identified and integrated into the nomogram. The concordance statistics were 0.84 and 0.74 in predicting the 5 -year DFS in the training and validation cohorts. The predictive performance of the nomograms outperformed the TNM model. Additionally, the patients were divided into two groups according to the nomogram score, and significant differences in OS and DFS were observed between the high risk and low risk groups. Finally, the role of postoperative treatments was evaluated based on the risk stratification; patients at high risk of disease recurrence showed an improvement in DFS after receiving postoperative treatments.

Conclusions: The nomogram showed good performance in predicting both OS and DFS in patients with PGC. It might be useful for selecting patients for postoperative treatments.

Keywords: Parotid gland cancer (PGC); nomogram; prognosis; survival

Submitted Apr 16, 2021. Accepted for publication Jul 12, 2021.

doi: $10.21037 / g s-21-250$

View this article at: https://dx.doi.org/10.21037/gs-21-250

\section{Introduction}

Primary parotid gland cancer (PGC) is an uncommon group of malignant tumor accounting for $14-25 \%$ of all parotid lesions and $3-6 \%$ of all head and neck malignancies (1). However, it primarily affects the major salivary glands, approximately $80 \%$ of which involves the parotid gland (2). With its relatively rare incidence and enormous diversity in histopathology types, prospective studies on parotid malignancies have traditionally been difficult to perform on a large scale, leading to a lack of widely accepted consensus 
regarding the epidemiology, management, and survival outcomes of PGC (3). Given the dearth of prospective evidence regarding $\mathrm{PGC}$, generalizable data on the outcomes and prognosis are largely based on the results of retrospective series. The important prognostic factors for parotid malignancies remain under debate.

Previous studies have identified several prognostic factors and developed prognostic tools that aid in clinical decisionmaking (4-10). However, many of these studies evaluated salivary gland cancer and did not specially focus on parotid cancer. Other previous studies used the data from public databases, such as SEER, of which many important clinical and tumor factors were missing. Moreover, some previous studies have suggested that PGC patients benefit from postoperative adjuvant treatments, but the role of adjuvant treatments remains controversial.

In this study, using a large sample size, we developed and validated two nomograms for predicting the longterm survival and disease recurrence by combining clinicopathological variables that influenced the prognosis of PGC. In addition, the value of postoperative treatments was evaluated in different risk groups based on the nomogram score.

We presented the following article in accordance with the TRIPOD Reporting Checklist (available at https:// dx.doi.org/10.21037/gs-21-250).

\section{Methods}

\section{Patient selection}

Data of patients with primary PGC were retrospectively collected and analyzed. Patients with primary PGC confirmed through pathological examination, who underwent surgery as initial management with or without postoperative treatments, and with complete clinicopathological, therapeutic and follow-up information were included in the study. Patients with recurrent or metastatic disease at the time of diagnosis, parotid lymphoma or sarcoma, parotid gland metastatic cancer from other primary sites, and secondary malignancy were excluded. The study was conducted in accordance with the Declaration of Helsinki (as revised in 2013). The study was approved by the Ethics Committee of the West China Hospital of Stomatology, Sichuan University (no. WCHSIRB-D-2019-712). Written informed consent was waived because of anonymous and retrospective data analysis.

Between 2000 and 2017, a total of 301 PGC patients diagnosed at West China Hospital of Stomatology, Sichuan
University were included into the entire cohort and randomly divided into the training cohort $(\mathrm{n}=210)$ and validation cohort ( $\mathrm{n}=91$ ) at a ratio of 7:3 (using sample function in $\mathrm{R}$ software) (Figure S1). The patients were followed-up every 3 months in the first 2 years, then every 4-6 months until 5 years, and annually thereafter. The median follow-up period was 61 months (range, 7-201 months).

\section{Predictor identification}

The survival endpoints included overall survival (OS) and disease-free survival (DFS). OS was calculated from the date of treatment to the date of death from any cause. DFS was calculated from the date of treatment to the date of disease relapse or death from any cause. Multivariate Cox regression analysis was conducted to identify the independent predictors of OS and DFS in the training cohort. The potential predictors with significance $(\mathrm{P}<0.05)$ in the univariate Cox regression analysis were included in the subsequent multivariate analysis (with a forward likelihood ratio method, $\mathrm{P}<0.05$ as the entry and $>0.10$ as the removal criteria).

\section{Nomogram construction and evaluation}

A nomogram model was established based on the results of the multivariate analysis. The concordance (C) index was used to measure the discriminative ability of the nomogram, and a calibration plot was drawn to reflect the agreement between the observed outcomes and predicted probabilities (11). A receiver operating characteristic (ROC) curve analysis was conducted to further evaluate the predictive performance of the nomogram for OS and DFS by calculating the area under the ROC curve (AUC). Timedependent ROC curve analysis was carried out to compare the abilities of the nomogram model and TNM model in predicting OS and DFS.

\section{Risk stratification and postoperative treatment comparison}

The sum score of each patient was computed based on the nomogram. The median score was used as the cut-off value for risk stratification. A Kaplan-Meier survival analysis with the log-rank test was performed to assess the significance of the prognostic difference between the low risk and the high risk groups. Subsequently, the clinical outcomes of the low risk and high risk patients who received different postoperative treatments were compared using the same method. 
Table 1 The demographic and clinicopathological characteristics of patients

\begin{tabular}{|c|c|c|}
\hline Characteristic & \multicolumn{2}{|c|}{ Training cohort $(n=210)$ Validation cohort $(n=91)$} \\
\hline \multicolumn{3}{|l|}{ Age, year } \\
\hline$<60$ & $160(76.2)$ & $69(75.8)$ \\
\hline$\geq 60$ & $50(23.8)$ & $22(24.2)$ \\
\hline \multicolumn{3}{|l|}{ Gender } \\
\hline Female & $108(51.4)$ & $46(50.5)$ \\
\hline Male & $102(48.6)$ & $45(49.5)$ \\
\hline \multicolumn{3}{|l|}{ Smoking history } \\
\hline No & $181(86.2)$ & $77(84.6)$ \\
\hline Yes & $29(13.8)$ & $14(15.4)$ \\
\hline \multicolumn{3}{|l|}{ Drinking history } \\
\hline No & $186(88.6)$ & $82(90.1)$ \\
\hline Yes & $24(11.4)$ & $9(9.9)$ \\
\hline \multicolumn{3}{|l|}{ Rapid growth } \\
\hline No & $169(80.5)$ & $71(78.0)$ \\
\hline Yes & $41(19.5)$ & $20(22.0)$ \\
\hline \multicolumn{3}{|l|}{ Pain } \\
\hline No & $150(71.4)$ & $65(71.4)$ \\
\hline Yes & $60(28.6)$ & $26(28.6)$ \\
\hline \multicolumn{3}{|l|}{ Facial palsy } \\
\hline No & 193 (91.9) & $81(89.0)$ \\
\hline Yes & $17(8.1)$ & $10(11.0)$ \\
\hline \multicolumn{3}{|l|}{ Grade } \\
\hline $\begin{array}{l}\text { Low/ } \\
\text { intermediate }\end{array}$ & 132 (62.9) & $61(67.0)$ \\
\hline High & $78(37.1)$ & 30 (33.0) \\
\hline \multicolumn{3}{|l|}{ pT stage } \\
\hline $1 / 2$ & 154 (73.3) & $73(80.2)$ \\
\hline $3 / 4$ & $56(26.7)$ & $18(19.8)$ \\
\hline \multicolumn{3}{|l|}{ pN stage } \\
\hline Negative & 195 (92.9) & 87 (95.6) \\
\hline Positive & $15(7.1)$ & $4(4.4)$ \\
\hline \multicolumn{3}{|l|}{ Neck dissection } \\
\hline No & $192(91.4)$ & $86(94.5)$ \\
\hline Yes & $18(8.6)$ & $5(5.5)$ \\
\hline
\end{tabular}

Table 1 (continued)
Table 1 (continued)

\begin{tabular}{lcc}
\hline Characteristic & Training cohort $(n=210)$ Validation cohort $(n=91)$ \\
\hline FN sacrifice & $134(63.8)$ & $65(71.4)$ \\
No & $76(36.2)$ & $26(28.6)$ \\
Yes & \\
Surgery margin & $190(90.5)$ & $87(95.6)$ \\
Negative & $20(9.5)$ & $4(4.4)$ \\
Positive/close &
\end{tabular}

Values presented as cases (\%). FN, facial nerve.

\section{Statistical analysis}

Statistical analysis was performed using SPSS (version 25.0, IBM Corporation, Armonk, NY) and R (version 3.6.1, R Foundation for Statistical Computing, Vienna, Austria). All $\mathrm{P}$ values in this study were two-sided, and a $\mathrm{P}$ value of $<0.05$ was considered significant.

\section{Results \\ Demographic and clinicopathological characteristics}

The demographic and clinicopathological characteristics of the patients in the training and validation cohorts are summarized in Table 1 . In the training cohort, $28.6 \%$ of the patients suffered pain, while $19.5 \%$ suffered rapid growth of the tumor. Postoperative findings showed that $73.3 \%$ of the patients had pT1/T2 stage tumors, while $92.9 \%$ had no lymph node metastasis. Of those patients, $62.9 \%$ had low- or intermediate-grade tumors, while $37.1 \%$ had high-grade tumors. Neck dissection was performed in $8.6 \%$ of the patients, while facial nerve (FN) sacrifice was performed in $36.2 \%$ of the patients. Positive/close margin was documented in $9.5 \%$ of the patients. In terms of treatment modalities in the entire cohort, 85 patients (28.2\%) underwent postoperative radiotherapy, 58 (19.3\%) underwent postoperative chemoradiotherapy and 31 $(10.3 \%)$ underwent postoperative chemotherapy.

\section{Identification of predictors in the training cohort}

The results of the univariate and multivariate Cox regression analyses based on the data from the training cohort are presented in Tables 2 and 3. Predictors with a $\mathrm{P}$ value of $<0.05$, as determined by univariate analysis, were 
Table 2 Univariate and multivariate analyses of OS in the training cohort

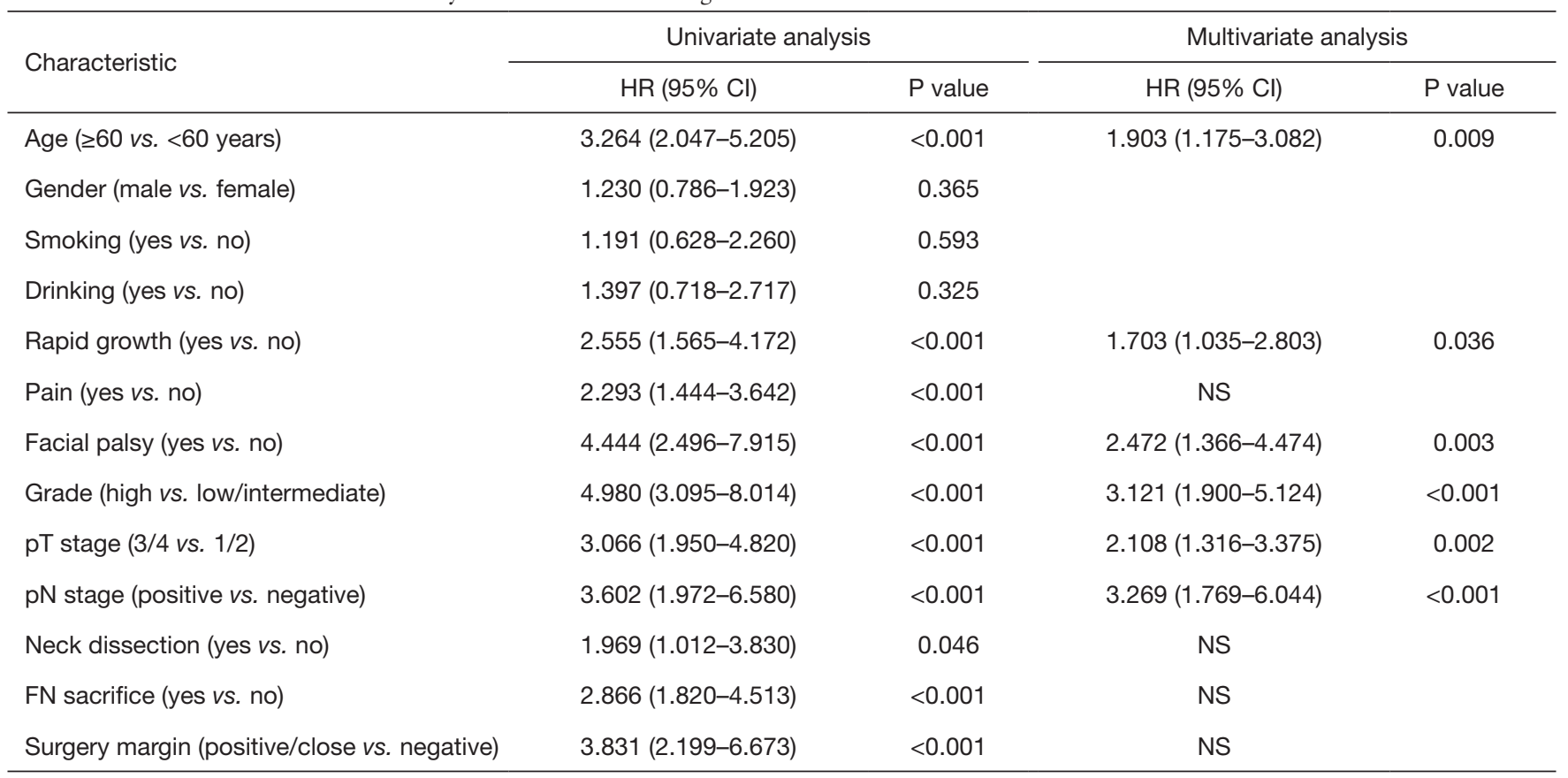

OS, overall survival; HR, hazard ratio; FN, facial nerve; NS, not significant.

included into multivariate analysis. Finally, multivariate analysis identified age, rapid growth, facial palsy, grade, $\mathrm{pT}$ stage, and $\mathrm{pN}$ stage as independent predictors of OS. Meanwhile, five indicators, including age, rapid growth, grade, FN sacrifice, and surgery margin, were significantly related to DFS.

\section{Development and validation of a nomogram for predicting $O S$}

The six independent risk factors of OS were integrated into an OS predicting nomogram (Figure 1). The C-index value of the nomogram in the training cohort was 0.797 [95\% confidence interval (CI): 0.753-0.841], and the calibration curve showed favorable consistency between the predicted probability of OS and the actual OS (Figure $2 A$ ). The AUCs of the nomogram for predicting OS at 3- and 5-year were 0.83 (95\% CI: $0.76-0.90$ ) and 0.84 (95\% CI: 0.78-0.90) (Figure 2B), which were higher than those of the TNM model (Figure $2 \mathrm{C}$ ). In the validation cohort, the $\mathrm{C}$-index value of the nomogram was 0.773 (95\% CI: 0.679-0.867), and the calibration curve also showed good agreement between the predicted and actual 3- and 5-year OS (Figure S2A). The AUCs of the nomogram were 0.84 (95\% CI: $0.67-1.00)$ and 0.78 (95\% CI: 0.65-0.91) in predicting the 3- and 5-year OS (Figure S2B), respectively. The performance of the nomogram was also preferable to that of the TNM model (Figure S2C).

\section{Development and validation of a nomogram for predicting DFS}

Five variables identified as independent indicators of DFS in the training cohort were used to construct a nomogram for predicting DFS (Figure 1). The C-index value of the nomogram in the training cohort was 0.727 (95\% CI: 0.677-0.776), and the calibration plot showed good agreement between the predicted probability of DFS and the actual DFS (Figure 2D). The AUCs of the nomogram score in predicting DFS at 3- and 5-year were 0.77 (95\% CI: $0.70-0.84$ ) and 0.84 (95\% CI: 0.78-0.90) (Figure 2E), which were higher than those of the TNM model (Figure $2 F$ ). Similar results were observed in the validation cohort. The C-index value of the nomogram was 0.698 (95\% CI: $0.627-$ 0.769 ), and the calibration plot also showed good agreement between the predicted and actual 3- and 5-year DFS (Figure S2D). The AUCs of the nomogram score were 0.75 (95\% CI: 0.64-0.86) and 0.74 (95\% CI: 0.63-0.85) in predicting the 3-and 5-year DFS (Figure S2E). The nomogram in the validation cohort also showed a better 
Table 3 Univariate and multivariate analyses of DFS in the training cohort

\begin{tabular}{|c|c|c|c|c|}
\hline Characteristic & \multicolumn{2}{|c|}{ Univariate analysis } & \multicolumn{2}{|c|}{ Multivariate analysis } \\
\hline Age ( $\geq 60$ vs. $<60$ years) & $2.395(1.565-3.664)$ & $<0.001$ & $1.809(1.166-2.808)$ & 0.008 \\
\hline Gender (male vs. female) & $1.309(0.881-1.943)$ & 0.182 & & \\
\hline Smoking (yes vs. no) & $0.932(0.509-1.706)$ & 0.819 & & \\
\hline Rapid growth (yes vs. no) & $1.945(1.238-3.056)$ & 0.004 & $1.651(1.032-2.642)$ & 0.037 \\
\hline Pain (yes vs. no) & $1.717(1.129-2.611)$ & 0.011 & NS & \\
\hline Facial palsy (yes vs. no) & $3.563(2.032-6.251)$ & $<0.001$ & NS & \\
\hline Grade (high vs. low/intermediate) & $3.142(2.096-4.710)$ & $<0.001$ & $2.045(1.322-3.163)$ & 0.001 \\
\hline Neck dissection (yes vs. no) & $1.905(1.041-3.486)$ & 0.037 & NS & \\
\hline FN sacrifice (yes vs. no) & $3.217(2.155-4.801)$ & $<0.001$ & $2.284(1.478-3.530)$ & $<0.001$ \\
\hline Surgery margin (positive/close vs. negative) & $3.333(1.960-5.666)$ & $<0.001$ & $1.853(1.060-3.240)$ & 0.031 \\
\hline
\end{tabular}

DFS, disease-free survival; HR, hazard ratio; FN, facial nerve; NS, not significant.
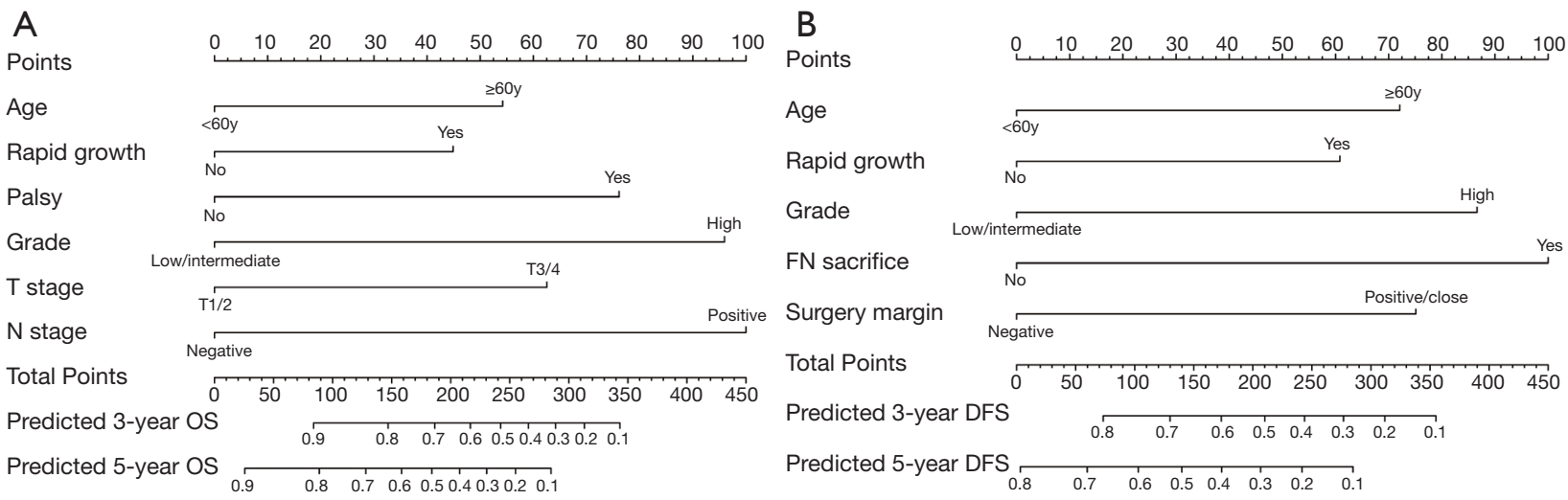

Figure 1 Prognostic nomograms for predicting the 3-and 5-year overall survival (OS) and disease-free survival (DFS) in patients with primary parotid gland cancer. (A) Prognostic nomogram for OS. (B) Prognostic nomogram for DFS.

performance compared with the TNM model (Figure S2F).

\section{Risk stratification and postoperative treatment comparison}

By applying the median value of the nomogram score calculated in the training cohort, patients from the three cohorts (training, validation, and entire cohort) were stratified into two separate groups: high risk and low risk. In each cohort, the low risk group had significantly better OS and DFS than the high risk group (all $\mathrm{P}<0.01$, Figure 3).

Based on risk stratification, we compared the benefits of different postoperative treatments in the entire cohort (Figure 4). Postoperative treatments did not prolong the survival of patients in the high risk and low risk groups. By contrast, postoperative chemoradiotherapy decreased the OS compared with surgery alone. In the high risk group, patients who received three postoperative treatments had higher DFS than those who did not. Similar results were 
A

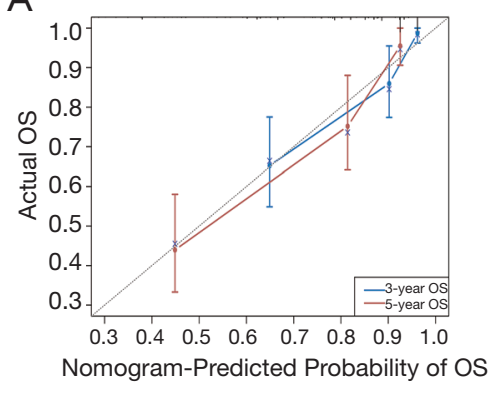

D

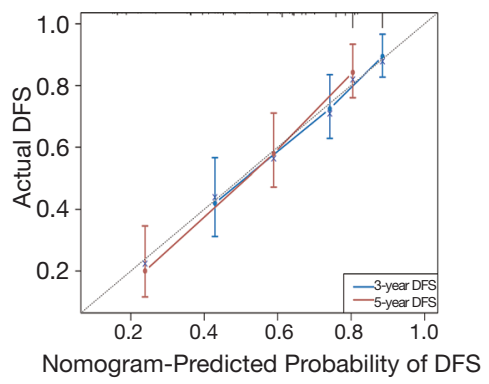

B

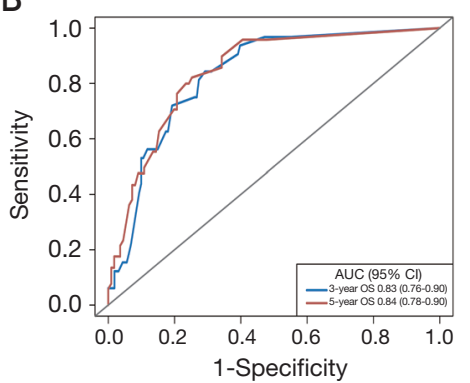

$\mathrm{E}$

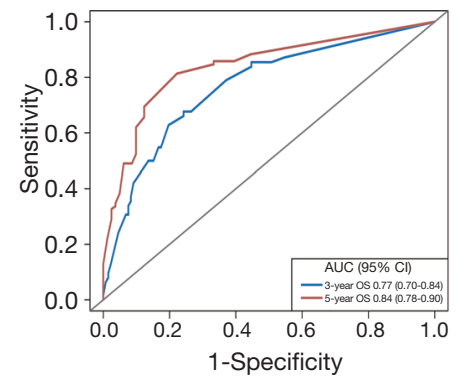

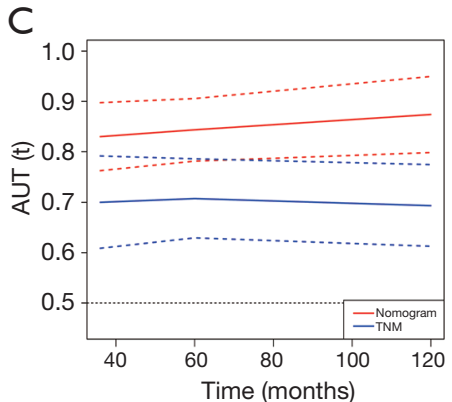

$\mathrm{F}$

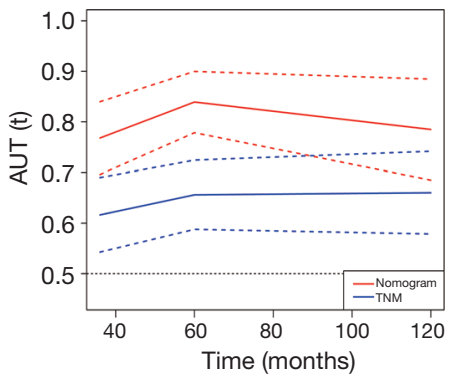

Figure 2 Performance of the prognostic nomograms in the training cohort. Calibration curves of the OS nomogram (A) and the DFS nomogram (D); ROC curves and AUCs of the OS nomogram (B) and the DFS nomogram (E); time-dependent ROC curves compared the OS nomogram and the TNM model (C) as well as compared the DFS nomogram and the TNM model (F).

found in the low risk group, except for postoperative chemoradiotherapy.

\section{Discussion}

Due to its remarkable heterogeneity and rarity, the risk stratification and prediction of oncological outcomes of PGC remains an unsolved challenge. Therefore, the development of comprehensive and applicable predicted tools is warranted to enable clinicians make effective clinical decisions. In the present study, we developed and validated two nomogram models that showed greater ability in predicting OS and DFS in patients with PGC after surgery. We also compared the different postoperative treatments based on risk stratification using the nomogram scores. These visualized nomogram models enable clinicians to estimate both the risk of disease recurrence and long-term survival in patients with PGC, which is also helpful in selecting patients for postoperative therapies.

PGC has been discussed in previous studies as a type of salivary gland cancer $(6,9,12-15)$. However, patients with PGC showed distinct clinical outcomes compared with those with non-parotid cancers (12). The present study specifically focused on PGC and included a larger sample size than most published studies except for those using the public database. The entire cohort was obtained from one of the largest stomatology hospitals in China, which possessed good generalizability and representativeness of patients with PGC. Based on the clinicopathological features of the population, the present study constructed two nomograms for predicting OS and DFS, and both showed greater discrimination and consistency in the training and validation cohorts. Furthermore, the addition of clinical signs and postoperative pathological features made the nomogram's performance more precise and preferable. Meanwhile, the identified predictive factors can be readily obtained for PGC patients who underwent surgery, making the nomogram feasible in clinical practice.

For patients with PGC, six independent risk factors, including age, rapid growth, facial palsy, grade, pT stage, and $\mathrm{pN}$ stage, were identified through univariate and multivariate analyses. A nomogram predicting OS was established based on the above predictors. The $\mathrm{C}$-index values calculated in the training cohort (0.797) and validation cohort (0.773) suggested that the nomogram performed superior discrimination for OS prediction. 

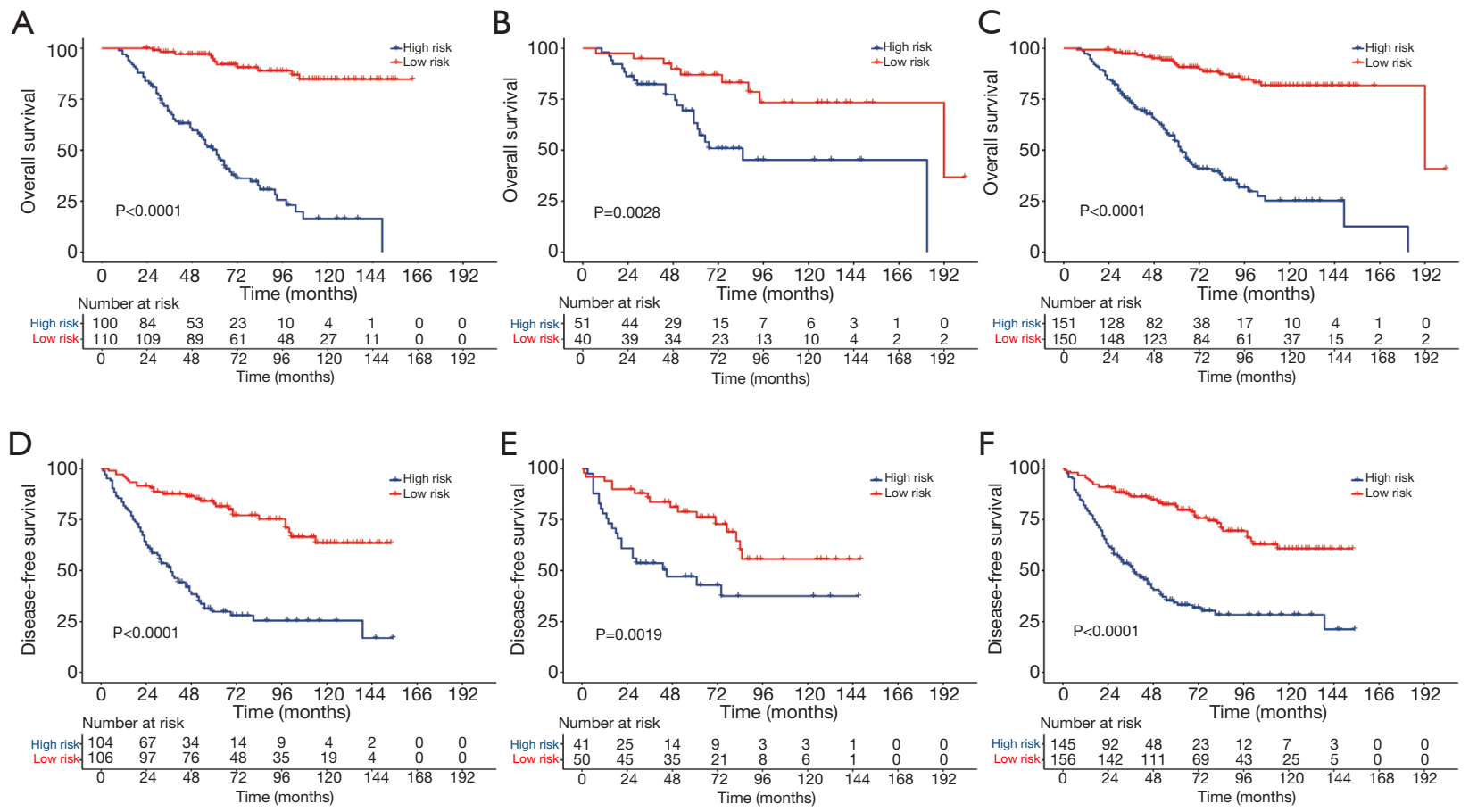

Figure 3 Kaplan-Meier curves for risk stratification. Kaplan-Meier curves of OS for risk stratification in the training cohort (A), the validation cohort (B) and the entire cohort (C); Kaplan-Meier curves of DFS for risk stratification in the training cohort (D), the validation cohort (E) and the entire cohort (F).

Meanwhile, the calibration curve showed good consistency between the nomogram predictions and the actual observations. It also showed better performance than the TNM model, which is currently the most commonly used model for predicting the clinical prognosis of PGC.

To date, several similar models have been used to predict individualized survival in patients with PGC. Ali et al. reported that 301 patients underwent surgery for salivary gland cancer. They identified five indicators including age, clinical T4 stage, grade, perineural invasion, and tumor size to generate a nomogram for predicting the OS (6). The nomogram had a preferable C-index value of 0.809 . However, patients with submandibular and sublingual gland cancers were also included in this study. Another study conducted by Mannelli et al. used six predictors, including age, histological grade, perineural invasion, pathological lymph node status, resection margins, and distant metastasis, to create a nomogram. But this nomogram was only verified using the internal validation technique of bootstrapping (4). Consistent with previously reported nomograms, we also identified that age, grade, tumor stage, and lymph node stage were predictive factors of OS. In addition, two invasive clinical signs, rapid growth and facial palsy, were incorporated into the model. FN dysfunction can be observed in up to $25 \%$ of patients with PGC and can predict worse disease outcomes (16). Terakedis et al. showed a $17 \%$ incidence of facial palsy in their cohort and demonstrated that clinical FN palsy was a risk factor for survival (2). Rapid tumor progression has also been proven to be significantly related to worse OS in patients with salivary duct carcinoma in the univariate analysis (17).

In the current study, a nomogram model for the assessment of the risk of disease recurrence after surgery was developed based on five variables, including age, rapid growth, grade, FN sacrifice, and surgery margin. The current nomogram performed well in predicting DFS, as supported by a $\mathrm{C}$ statistic of 0.727 in the training cohort. Its good performance was also confirmed in the validation cohort, with a $\mathrm{C}$ statistic of 0.698 . Compared with the TNM model, the present nomogram showed greater discriminatory ability in DFS, suggesting its reliability for evaluating recurrence in patients with PGC.

Furthermore, some previous studies have constructed similar recurrence-predicted nomograms. Vander Poorten 

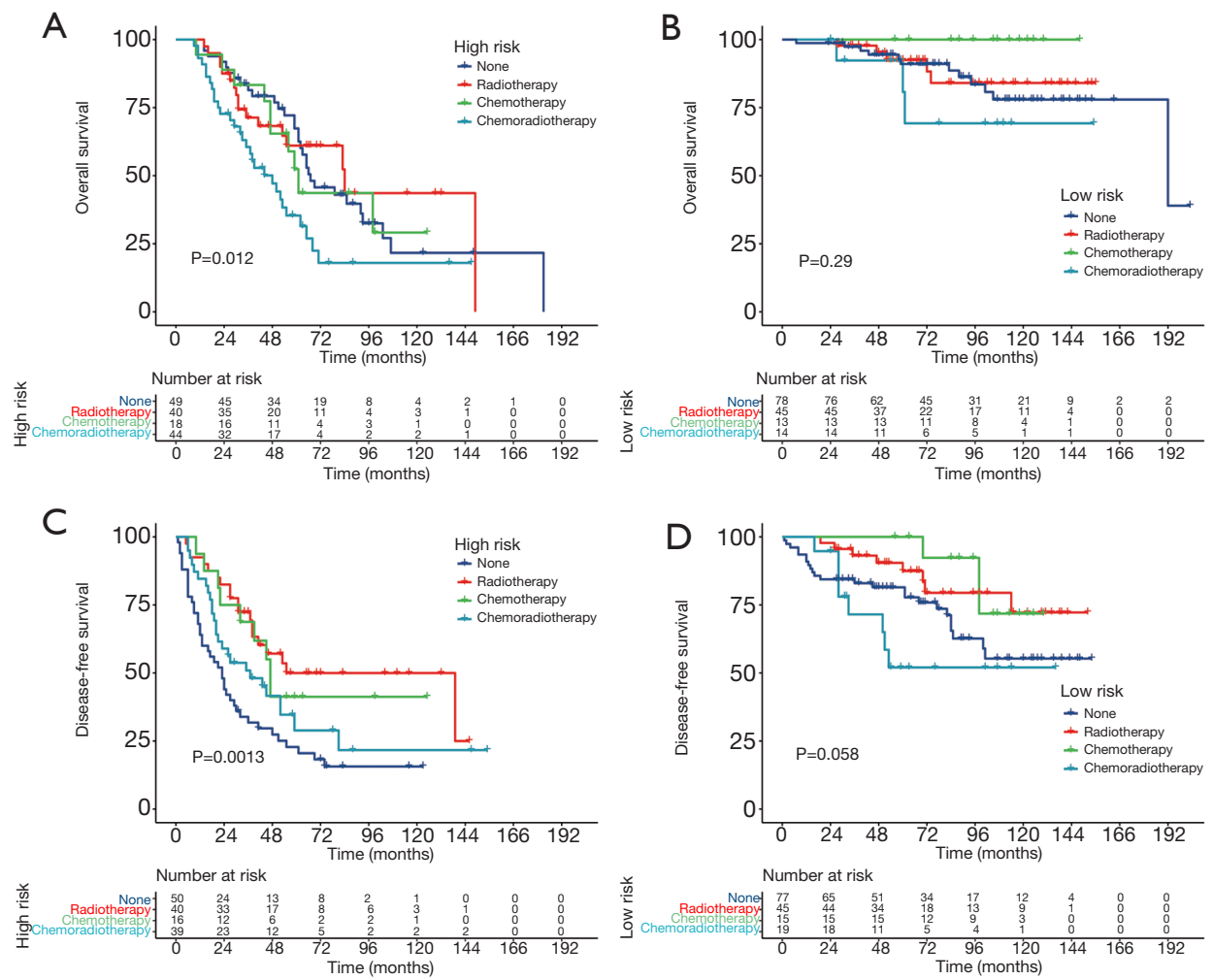

Figure 4 Kaplan-Meier curves of postoperative treatments for risk stratification. Kaplan-Meier curves of postoperative treatments in the high risk group (A) and the low risk group (B) in terms of OS; Kaplan-Meier curves of postoperative treatments in the high risk group (C) and the low risk group (D) in terms of DFS.

et al. (18) first presented a prognostic model for predicting the relapse risk of patients with parotid carcinoma after surgery. In this model, age, clinical T stage, clinical N stage, pain, skin invasion, FN dysfunction, perineural growth, and positive surgical margins were identified as predictors for tumor recurrence and incorporated into the pre- and postoperative nomograms. The model's performance was subsequently validated in a series of internal and external cohorts $(5,19,20)$. In the present study, except for the predictors identified in other studies, we also revealed that rapid growth was significantly associated with DFS. Similar results were also reported in another study (17).

Postoperative risk prediction of clinical outcomes has the potential to select eligible candidates for adjuvant treatments. Surgery remains the cornerstone of managing PGC, which could be generally used to cure low-stage, low-grade tumors (21), but is not sufficient for patients with high-risk features $(22,23)$. Postoperative radiotherapy improved locoregional control and survival for selected patients with poor prognostic features (24-26). Besides, systemic treatment has traditionally been used for metastatic or recurrent disease, while postoperative chemotherapy has rarely been studied (27). Postoperative chemoradiotherapy, a more intensive adjuvant therapy, did not improve the survival rates in previous studies $(28,29)$.

This study also attempted to discuss the benefits of postoperative therapies in different risk strata. First, according to the established nomogram score, patients were divided into a high risk group and a low risk group. The low risk group exhibited significantly better OS and DFS than the high risk group in the training, validation, and entire cohorts. Survival analysis revealed that adjuvant treatments could significantly increase the DFS in patients with high risk of disease recurrence. Patients categorized in the low risk group who underwent postoperative radiotherapy or chemotherapy also had a higher DFS than those who underwent surgery alone. However, when comparing postoperative treatments with surgery alone, no OS benefits were observed, regardless of whether the patients were at high risk or at low risk. Unfortunately, 
surgery followed by chemoradiotherapy aggravated the oncological outcomes, which could be explained by the fact that chemoradiotherapy is mainly used to treat patients with more adverse factors. Overall, with the improvement in disease control, patients categorized in the group at high risk of recurrence could be considered ideal candidates for postoperative treatments, and postoperative radiotherapy or chemotherapy was also optional for patients at low risk of recurrence.

The present study has several limitations. First, the current nomogram model was derived based on a population from a single institution. Although the analysis of the validation cohort showed that the nomogram also performed well, the results need to be verified in the external cohort. Second, this was a retrospective study in which selection bias was unavoidable. However, bias was minimized by including a large cohort of consecutive patients. In addition, a longer follow-up time is required, especially for patients with some slow-growing pathological types.

\section{Conclusions}

In conclusion, the current nomogram models showed good performance in the prediction of disease recurrence and long-term survival in patients with PGC who underwent surgery. It could provide reliable information about the oncological outcomes of these patient populations. The application of the nomogram models in risk stratification could facilitate clinicians in selecting suitable candidates for postoperative treatments.

\section{Acknowledgments}

Funding: This work was supported by the National Natural Science Foundation of China [grant number: 81672386].

\section{Footnote}

Reporting Checklist: The authors have completed the TRIPOD reporting checklist. Available at https://dx.doi. org/10.21037/gs-21-250

Data Sharing Statement: Available at https://dx.doi. org/10.21037/gs-21-250

Conflicts of Interest: All authors have completed the ICMJE uniform disclosure form (available at https://dx.doi. org/10.21037/gs-21-250). The authors have no conflicts of interest to declare.

Ethical Statement: The authors are accountable for all aspects of the work in ensuring that questions related to the accuracy or integrity of any part of the work are appropriately investigated and resolved. The study was conducted in accordance with the Declaration of Helsinki (as revised in 2013). The study was approved by the Ethics Committee of the West China Hospital of Stomatology, Sichuan University (No. WCHSIRB-D-2019-712) and individual consent for this retrospective analysis was waived.

Open Access Statement: This is an Open Access article distributed in accordance with the Creative Commons Attribution-NonCommercial-NoDerivs 4.0 International License (CC BY-NC-ND 4.0), which permits the noncommercial replication and distribution of the article with the strict proviso that no changes or edits are made and the original work is properly cited (including links to both the formal publication through the relevant DOI and the license). See: https://creativecommons.org/licenses/by-nc-nd/4.0/.

\section{References}

1. Zbären P, Schüpbach J, Nuyens M, et al. Carcinoma of the parotid gland. Am J Surg 2003;186:57-62.

2. Terakedis BE, Hunt JP, Buchmann LO, et al. The Prognostic Significance of Facial Nerve Involvement in Carcinomas of the Parotid Gland. Am J Clin Oncol 2017;40:323-8.

3. Davies L, Welch HG. Epidemiology of head and neck cancer in the United States. Otolaryngol Head Neck Surg 2006;135:451-7.

4. Mannelli G, Alessandro F, Martina F, et al. Nomograms predictive for oncological outcomes in malignant parotid tumours: recurrence and mortality rates of 228 patients from a single institution. Eur Arch Otorhinolaryngol 2019. [Epub ahead of print]. doi: 10.1007/s00405-019-05556-9.

5. Peeperkorn S, Meulemans J, Van Lierde C, et al. Validated Prognostic Nomograms for Patients With Parotid Carcinoma Predicting 2- and 5-Year Tumor RecurrenceFree Interval Probability. Front Oncol 2020;10:1535.

6. Ali S, Palmer FL, Yu C, et al. Postoperative nomograms predictive of survival after surgical management of malignant tumors of the major salivary glands. Ann Surg Oncol 2014;21:637-42. 
7. Hu JQ, Yu PC, Shi X, et al. Prognostic Nomograms for Predicting Overall Survival and Cancer-Specific Survival of Patients with Major Salivary Gland Mucoepidermoid Carcinoma. J Cancer 2019;10:4380-8.

8. Sun J, Sun Y, Yang F, et al. Nomogram to predict the prognosis of parotid gland mucoepidermoid carcinoma: a population-based study of 1306 cases. PeerJ 2019;7:e7237.

9. Lu CH, Liu CT, Chang PH, et al. Develop and validation a nomogram to predict the recurrent probability in patients with major salivary gland cancer. J Cancer 2017;8:2247-55.

10. Hu YH, Li W, Zhang CY, et al. Prognostic nomogram for disease-specific survival of carcinoma ex pleomorphic adenoma of the salivary gland. Head Neck 2017;39:2416-24.

11. Steyerberg EW, Vergouwe Y. Towards better clinical prediction models: seven steps for development and an ABCD for validation. Eur Heart J 2014;35:1925-31.

12. Nam SJ, Roh JL, Cho KJ, et al. Risk Factors and Survival Associated with Distant Metastasis in Patients with Carcinoma of the Salivary Gland. Ann Surg Oncol 2016;23:4376-83.

13. Holtzman A, Morris CG, Amdur RJ, et al. Outcomes after primary or adjuvant radiotherapy for salivary gland carcinoma. Acta Oncol 2017;56:484-9.

14. Ali S, Palmer FL, Yu C, et al. A predictive nomogram for recurrence of carcinoma of the major salivary glands. JAMA Otolaryngol Head Neck Surg 2013;139:698-705.

15. Hay A, Migliacci J, Zanoni DK, et al. Validation of nomograms for overall survival, cancer-specific survival, and recurrence in carcinoma of the major salivary glands. Head Neck 2018;40:1008-15.

16. Wierzbicka M, Kopeć T, Szyfter W, et al. The presence of facial nerve weakness on diagnosis of a parotid gland malignant process. Eur Arch Otorhinolaryngol 2012;269:1177-82.

17. Otsuka K, Imanishi Y, Tada Y, et al. Clinical Outcomes and Prognostic Factors for Salivary Duct Carcinoma: A Multi-Institutional Analysis of 141 Patients. Ann Surg Oncol 2016;23:2038-45.

18. Vander Poorten VL, Balm AJ, Hilgers FJ, et al. The development of a prognostic score for patients with parotid carcinoma. Cancer 1999;85:2057-67.

19. Vander Poorten VL, Hart AA, van der Laan BF, et al. Prognostic index for patients with parotid carcinoma: external validation using the nationwide 1985-1994 Dutch Head and Neck Oncology Cooperative Group database.
Cancer 2003;97:1453-63.

20. Poorten VV, Hart A, Vauterin T, et al. Prognostic index for patients with parotid carcinoma: international external validation in a Belgian-German database. Cancer 2009; 115:540-50.

21. Lewis AG, Tong T, Maghami E. Diagnosis and Management of Malignant Salivary Gland Tumors of the Parotid Gland. Otolaryngol Clin North Am 2016;49:343-80.

22. Chandana SR, Conley BA. Salivary gland cancers: current treatments, molecular characteristics and new therapies. Expert Rev Anticancer Ther 2008;8:645-52.

23. Laurie SA, Licitra L. Systemic therapy in the palliative management of advanced salivary gland cancers. J Clin Oncol 2006;24:2673-8.

24. Terhaard CH. Postoperative and primary radiotherapy for salivary gland carcinomas: indications, techniques, and results. Int J Radiat Oncol Biol Phys 2007;69:S52-5.

25. Terhaard CH, Lubsen H, Rasch CR, et al. The role of radiotherapy in the treatment of malignant salivary gland tumors. Int J Radiat Oncol Biol Phys 2005;61:103-11.

26. Mahmood U, Koshy M, Goloubeva O, et al. Adjuvant radiation therapy for high-grade and/or locally advanced major salivary gland tumors. Arch Otolaryngol Head Neck Surg 2011;137:1025-30.

27. Rizk S, Robert A, Vandenhooft A, et al. Activity of chemotherapy in the palliative treatment of salivary gland tumors: review of the literature. Eur Arch Otorhinolaryngol 2007;264:587-94.

28. Gebhardt BJ, Ohr JP, Ferris RL, et al. Concurrent Chemoradiotherapy in the Adjuvant Treatment of Highrisk Primary Salivary Gland Malignancies. Am J Clin Oncol 2018;41:888-93.

29. Amini A, Waxweiler TV, Brower JV, et al. Association of Adjuvant Chemoradiotherapy vs. Radiotherapy Alone With Survival in Patients With Resected Major Salivary Gland Carcinoma: Data From the National Cancer Data Base. JAMA Otolaryngol Head Neck Surg 2016;142:1100-10.

Cite this article as: Wei Z, Zhang Z, Yang L, He L, Liu Z, He Y, Wang J, Mu X, Li R, Huang Y, Peng X. Development and validation of nomograms for predicting survival and recurrence in patients with parotid gland cancer. Gland Surg 2021;10(8):23982407. doi: $10.21037 / g s-21-250$ 


\section{Supplementary}

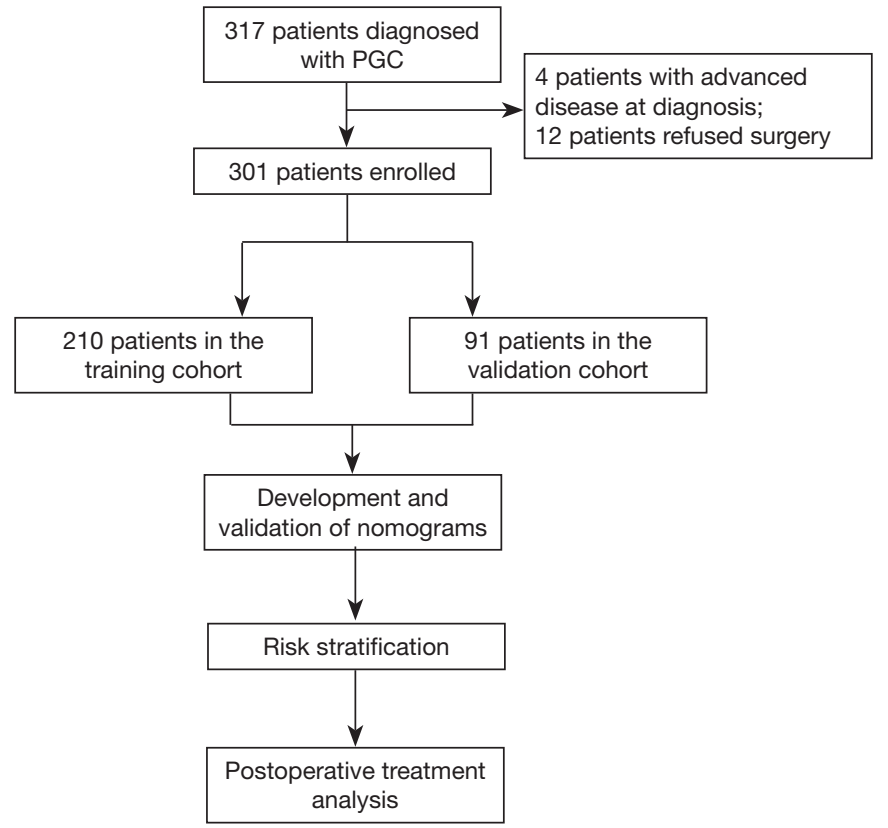

Figure S1 Flow chart of the study process.
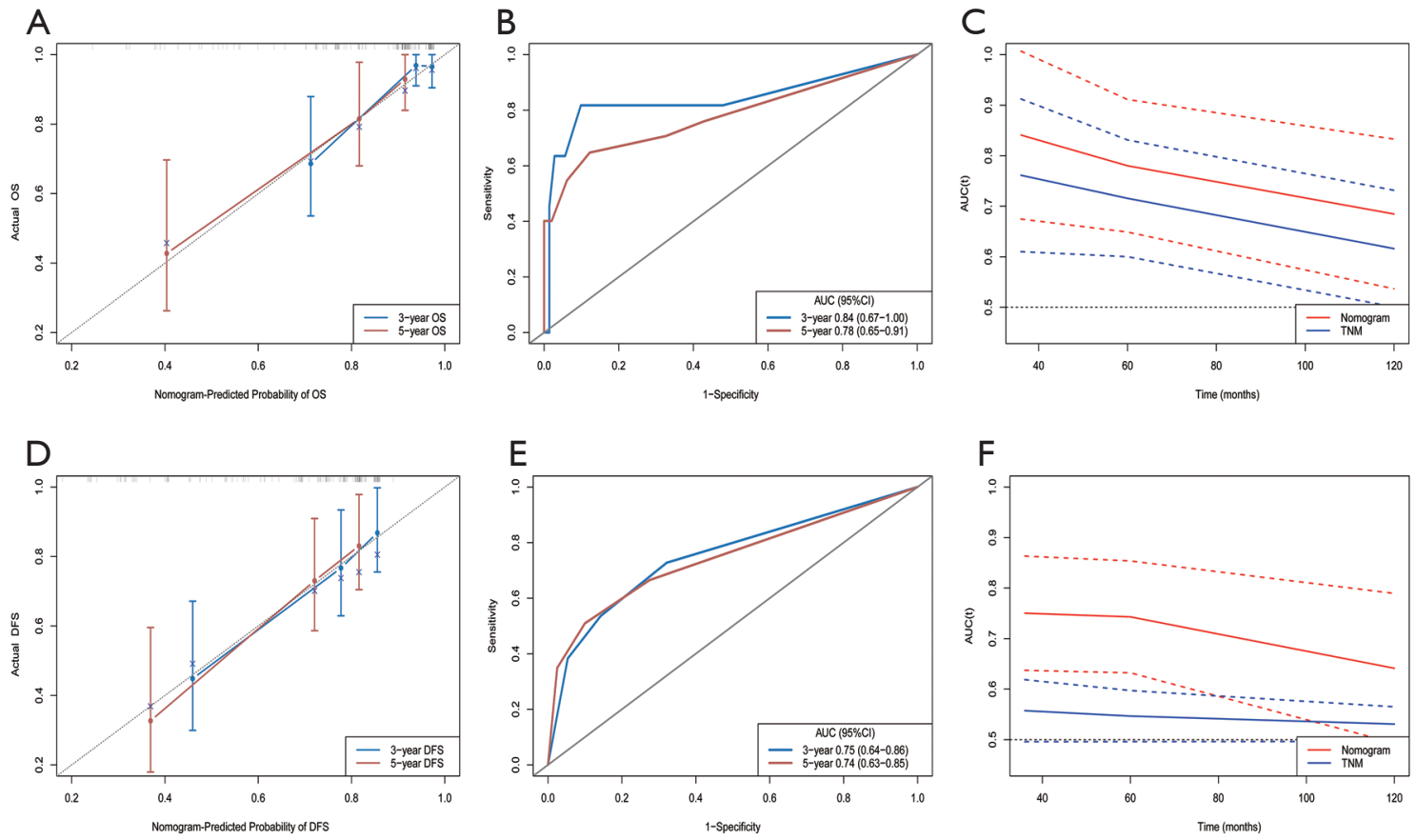

Figure S2 The performance of the prognostic nomograms in the validation cohort. Calibration curves of the OS nomogram (A) and the DFS nomogram (D); ROC curves and AUCs of the OS nomogram (B) and the DFS nomogram (E); time-dependent ROC curves compared the OS nomogram and the TNM model (C) as well as compared the DFS nomogram and the TNM model (F). 